ISSN: 0213-1854

\title{
La terminología vitivinícola en The Winemaker y su traducción al español y al alemán
}

\section{(The translation of viticulture and viniculture terminology in The Winemaker into Spanish and German)}

\author{
MARÍA PILAR CASTILLO BERNAL \\ pilar.castillo.bernal@uco.es \\ Universidad de Córdoba
}

Fecha de recepción: 23 de agosto de 2017

Fecha de aceptación: 30 de septiembre de 2017

Resumen: El presente trabajo se centra en la novela La bodega, del autor estadounidense Noah Gordon, más conocido por el éxito de su obra El médico. El interés de los lectores alemanes y españoles por Gordon justifica la elección de una novela ambientada en el siglo XIX en los viñedos catalanes. El objeto principal de nuestro análisis es el lenguaje especializado de la vid y el vino y la traducción del mismo. Para llevarlo a cabo, se ha realizado una extracción terminológica en las versiones inglesa, alemana y española de la novela. Los términos y fragmentos extraídos se presentan clasificados por campo temático, categoría gramatical y repercusión en la novela. Del mismo modo, los paratextos publicados con la misma se han estudiado para completar un estudio anterior sobre este mismo objeto.

Palabras clave: Lenguaje del vino. Lenguaje especializado. Traducción literaria.

\begin{abstract}
This paper focuses on the novel The Winemaker, by American author Noah Gordon, who is best known for the success of his bestseller The Physician. The interest of Spanish and German readers in Gordon justifies this analysis of a novel which is set in the $19^{\text {th }}$ century in the Catalonian vineyards. The main purpose of our analysis is the specialised language of vines and wine and the translation thereof. For this, a terminology search has been conducted in the English, Spanish and German versions of the novel. The terms and excerpts are classified according to thematic fields, grammar categories and purposes served in the novel. Likewise, the paratexts accompanying the novel have been studied in order to complete previous investigations on this very same object.
\end{abstract}

Key words: Language of wine. Specialised language. Literary translation. 
María Pilar CASTillo Bernal

\section{Introducción}

El interés por el mundo del vino y, más concretamente, por la terminología del mismo, no sorprende teniendo en cuenta el importante componente cultural y económico de este producto en nuestro país, especialmente en lo que respecta a la exportación e internacionalización. En los últimos años, se han realizado esfuerzos tanto desde las instituciones como desde el sector privado para promocionar el sector vitivinícola español en el ámbito nacional e internacional; muestra de ello son las sucesivas ediciones de FENAVIN, la Feria Nacional del Vino, o la Cumbre Internacional del Vino en sus sendas ediciones de 2013 y 2015. Desde el punto de vista de la investigación traductológica, el lenguaje de la vid y el vino ha sido objeto de estudio por parte del grupo GIRTraduvino, de la Universidad de Valladolid, que ha dado lugar a sendas publicaciones en 2006 y 2010. ${ }^{1}$ Más recientemente, desde la Universidad de Córdoba, encontramos el volumen dedicado a Lenguajes especializados y traducción: Vitivinicultura (Balbuena Torezano y Álvarez Jurado, 2015). Esta obra constituye un trabajo preliminar al que posteriormente se ha constituido como Proyecto I+D+i FFI 2016-79785-R Weinapp: sistema multilingüe de información y recursos vitivinicolas, financiado por el Ministerio de Economía y Competitividad.

El presente estudio se enmarca en una línea de investigación sobre el lenguaje de especialidad en el texto literario, con base en un enfoque integrador respecto de la presencia de la terminología vitivinícola en textos de la más diversa índole. No en vano, el texto literario promueve la consolidación y difusión de expresiones y términos como paremias o refranes. No obstante, el trabajo que presentamos no se centra en estas manifestaciones lingüísticas tal como se han estudiado hasta la fecha (véase el apartado 2), sino en el tratamiento de la terminología de especialidad en un texto en principio no especializado como es la novela. Del mismo modo, la importancia de la literatura de ficción a la hora de promover los aspectos culturales de un país o región —o, en este caso, un producto gastronómico- quedará patente en las conclusiones de este trabajo.

\section{Antecedentes y contextualización de la obra}

No son pocos los autores que han estudiado la presencia del vino en la literatura, entre los que cabe destacar la obra de Muro (2006). Desde el punto de vista lingüístico, diversos autores se centran en las paremias en torno al vino presentes en la lengua (Forgas i Berdet, 1996; Corbacho, 2004; Castillo, 2012;

1 Vid. Ibáñez et al. 2006, 2010 
La terminología vitivinícola en The Winemaker y su traducción...

Martínez Fraile, 2015). A su vez, en el contexto del grupo GIRTraduvino, encontramos algunos trabajos en torno a la presencia del vino en la literatura y su traducción, como el estudio de Nord (2010). Destacaremos especialmente el artículo de Zarandona (2010), por la proximidad temática con el presente estudio.

En su contribución "Novelas populares norteamericanas ambientadas en viñedos y bodegas y algunas reflexiones de traducción", Zarandona examina doce novelas en cuanto a las circunstancias de su publicación, temática y traducción al español. Por último, se centra en La bodega, de Noah Gordon, de la que realiza un análisis más exhaustivo en cuanto al éxito editorial de la misma, así como un análisis comparativo de extractos de la versión española y catalana. Ante la imposibilidad de localizar un supuesto original en lengua inglesa, Zarandona presenta dos hipótesis: que el original no llegara a publicarse por razones comerciales o que se trate de pseudotraducciones, ya que las coincidencias lingüísticas entre ambas versiones sugieren que se trata de una traducción del español al catalán, o viceversa, sin que existiera el supuesto original inglés:

Noah Gordon no es $\mathrm{al}^{2}$ autor, ni del texto catalán ni del
castellano y únicamente habría prestado su nombre, como
si de una marca comercial se tratara. (...) En el caso de que
una de las dos obras fuera el original y la otra su
traducción, parece mucho más probable que El celler sea el
original y La bodega su traducción: por tema, geografía,
personajes, costumbres y todo tipo de referencias
culturales catalanas, y por la constante presencia de
catalanismos lingüísticos y sintácticos (calcos) de la
versión castellana (Zarandona, 2010: 223 )

En nuestro estudio, incluiremos no obstante el texto inglés publicado por Barcelona Digital Editions en 2012 con el título The Winemaker, ya que el foco de interés en este caso es el tratamiento de la terminología vitivinícola en los tres idiomas, aunque no sea posible establecer con seguridad si realmente el texto inglés es el original.

\subsection{Contextualización de la novela}

La acción de La bodega comienza en Languedoc, Francia, a finales del s.

2 Sic. 


\section{MARía Pilar CASTILLO BERNAL}

XIX, cuando el protagonista Josep Álvarez decide volver a su Cataluña natal al recibir la noticia del fallecimiento de su padre. Allí se hará cargo del viñedo familiar, mientras que en sucesivas analepsis se revela que se vio obligado a huir a Francia tras su implicación en las guerras carlistas y en el asesinato del general Juan Prim.

Se trata de la novela más reciente de Noah Gordon, autor estadounidense cuyo mayor éxito editorial es El médico, publicada en 1986. No es la primera obra en la que el escritor manifiesta un interés por España: ya su novela $E l$ último judío, publicada en 2000, transcurre en nuestro país en el s. XV, en la época de la expulsión de los judíos ${ }^{3}$. En los agradecimientos al final de la versión española de La bodega, el autor indica lo siguiente:

Este libro es mi carta de amor a un país. No descubrí las glorias del buen vino hasta que, siendo ya un hombre de mediana edad, empecé a viajar a España, donde pronto desarrollé un profundo afecto por la gente, su cultura y sus vinos. Cuando decidí escribir una historia sobre ellos, escogí centrarme en la mitad del siglo XIX porque fue el período de la plaga de la filoxera y de las Guerras Carlistas. Ubiqué mi viña ficticia en el Penedès porque, viviendo allí, mi protagonista tenía acceso al mismo tiempo a Barcelona y a las regiones vinícolas del sur de Francia (Gordon, 2007)

Es de destacar que la versión en inglés presenta una versión adaptada de los agradecimientos (Acknowledgments), fechada el 23 de abril de 2012, donde se exponen las siguientes consideraciones sobre los títulos de la novela en inglés y alemán:

This novel has been a bestseller in a number of countries under the title of The Bodega, except in Germany, where with my permission the book was called The Catalan. While Europeans are familiar with the concept that a bodega is a place where wine is made and sold, in America it has become a term to describe a small grocery business. To avoid that confusion, this novel is published under the

${ }^{3}$ Ver la sinopsis de la obra en la web oficial de la editorial. Disponible en línea en: http://www.rocalibros.com/roca-editorial/catalogo/Noah+Gordon/El+ultimo+judio [Fecha de consulta: 9 de agosto 2017] 
La terminología vitivinícola en The Winemaker y su traducción...

title of The Winemaker. I am happy to offer my story in the English language to each reader with affection and respect (Gordon, 2012)

En el mismo párrafo al final de los agradecimientos de la versión española encontramos menciones a la editorial Roca Libros y a sus editores, junto a una mención al traductor: "Enrique de Hériz, a quien conocí por primera vez como intérprete y más adelante como director editorial, y que hoy se ha convertido en escritor premiado, me hizo el honor de traducir este libro del inglés original" (Gordon, 2007). Estos datos parecen contradecir la segunda hipótesis de Zarandona (que no existiera un original en lengua inglesa) y confirmar parcialmente la primera: que, por razones comerciales, la novela se publicó inicialmente en español y catalán, siendo editada posteriormente en su versión en inglés.

\subsection{El lenguaje de especialidad en la literatura}

El propósito principal de este trabajo es analizar el lenguaje de especialidad en el texto literario. Siguiendo a Hoffmann, entendemos el lenguaje de especialidad como:

(...) die Gesamtheit aller sprachlichen Mittel, die in einem fachlich begrenzbaren Kommunikationsbereich verwendet werden, um die Verständigung zwischen den in diesem Bereich tätigen Menschen zu gewährleisten (Hoffmann, 1985: 53, apud Sandrini, 1996: 13)

Por tanto, en el contexto del presente trabajo el lenguaje de especialidad que nos interesa es el empleado por viticultores y vinicultores, tal como corresponde a la temática de la novela, si bien fuera de su ámbito habitual de aplicación y dirigido al lector lego. El uso del lenguaje de especialidad en la literatura y su tratamiento por parte del traductor está documentado en trabajos anteriores, tales como Escobar (2005), Solana (2005) o Santana y Fortea (2013). Estos últimos, citando a Wienen, enumeran las siguientes funciones que puede cumplir el uso de este lenguaje en el texto literario:

- Caracterizar a los personajes y su día a día

-Producir efectos humorísticos, irónicos o paródicos

-Favorecer la identificación de los personajes entre sí/con

el lector

-Divulgar el conocimiento científico 
María Pilar Castillo Bernal

-Producir un efecto poético

-Crear tensión narrativa (Wienen, 2011: 818, citado por

Santana y Fortea, 2013: 137)

A estas podemos añadir, en el texto que nos ocupa, la promoción de una determinada actividad o región desde un punto de vista poético o literario, como es el caso de las explotaciones vitivinícolas catalanas que son objeto de interés en La bodega. En efecto, el autor expone en los agradecimientos detalles de sus visitas a diversas bodegas catalanas. También hace mención a su nuera, española a juzgar por el nombre y la descripción proporcionada ${ }^{4}$ :

Mi nuera, Maria Palma Castillón, nunca rehuyó la investigación de un dato y le agradezco, así como al Centre de Promoció de la Cultura Popular i Tradicional Catalana, en Barcelona, las respuestas a cuantas preguntas ella les hizo en mi nombre, que podían versar sobre las campanadas de una iglesia a la práctica de contratar plañideras (Gordon, 2007)

Todo esto indica un interés personal y autobiográfico de Gordon hacia el ámbito vitivinícola, en concreto las regiones catalanas en las que se ambienta la novela, lo cual justificaría el uso del lenguaje de especialidad y las extensas descripciones sobre vitivinicultura que se encuentran a lo largo de toda la novela. El mismo autor destaca en su web oficial la importancia de sus viajes a nuestro país en el proceso de documentación de la novela: "I wrote The Physician using only library research. For my last two books, The Last Jew and The Winemaker, I was fortunate enough to be able to make several trips to Spain ${ }^{5 "}$.

\section{Metodología}

La elección de La bodega para el presente estudio se debe principalmente a la

\footnotetext{
${ }^{4}$ Véase asimismo los agradecimientos en El último judío, donde se menciona a su nuera y su hijo como residentes en Cataluña: "Mi nuera María Palma Castillón tradujo, interpretó y leyó las pruebas de la edición española y, siempre que estábamos en el mismo país, nos atiborraba con excelentes platos de la cocina catalana. Mi hijo Michael Seay Gordon estuvo constantemente a mi lado con informes, recortes de periódico, llamadas telefónicas, consejos, inteligencia y apoyo. Entrevistó a muchas personas en mi nombre y fue un inmejorable compañero durante varios de mis viajes a España" (Gordon, 2011)

${ }^{5}$ Disponible en línea en: http://www.noahgordonbooks.com/noahstory.htm [Fecha de consulta: 9 de agosto 2017]
} 
La terminología vitivinícola en The Winemaker y su traducción...

popularidad del autor en España y Alemania, nuestros principales ámbitos lingüísticos de interés y en los que se produjo todo un fenómeno de ventas de la novela El médico que condicionó la posterior recepción de Gordon en Europa ${ }^{6}$. Por ello, además de la versión inglesa — presumiblemente, el texto original_ publicada en 2012 por Barcelona Digital Editions, S.L., se analizarán la versión española La bodega, traducida por Enrique de Hériz, y la versión alemana Der Katalane, traducida por Klaus Berr ${ }^{7}$.

En el tratamiento de las tres versiones, seguiremos los siguientes parámetros de análisis del lenguaje de especialidad en un texto dado establecidos por Wienen:

\begin{abstract}
-Densidad terminológica (en forma de lexemas de especialidad aislados / fragmentos / capítulos enteros, etc.) -Explicación de los términos especializados (mediante notas a pie de página / paráfrasis a cargo del narrador / explicación a través de otros personajes, etc.)

-Estrategias para introducir el lenguaje de especialidad (sin previo aviso / con estrategias de focalización, p. ej. a través de un discurso ficticio, etc.)

-Situación narrativa (uso del lenguaje de especialidad por parte del narrador / un personaje; experto / lego; en forma de diálogo / monólogo, etc.) (Wienen, 2011: 818, citada por Santana y Fortea, 2013: 137)
\end{abstract}

Cabe destacar que la terminología vitivinícola está presente a lo largo de toda la novela, por lo que esta se ha analizado en su totalidad, sin seleccionar partes o capítulos concretos. Se realizó un vaciado terminológico manual, partiendo del texto en español, y se procedió a la comparación con las versiones en inglés y alemán. Se analizaron un total de 284 unidades, incluyendo terminología, fraseología y culturemas específicos tal como se desglosará en el siguiente apartado. Los paratextos de las distintas versiones han sido asimismo incluidos en el análisis.

${ }^{6}$ Ibid.: “( ...) a publisher from Germany named Karl H. Blessing. He read the book in New York, loved it, and bought it. He made certain that every clerk in every book store in Germany received a reading copy, and the result was a publishing phenomenon in that country, where sales of The Physician have topped eight million copies. At the same time, a similar phenomenon was occurring in Spain, and as news from both these countries reached publishers all over Europe, they flocked to buy the book."

${ }^{7}$ En ambas ediciones se indica que la obra original se titula The Bodega. 
María Pilar Castillo Bernal

\section{Análisis terminológico y traductológico}

Respecto a la macroestructura de la novela, esta se compone de 62 capítulos divididos en cinco partes: "El regreso", "El grupo de cazadores", "En el mundo", "La tierra de los Álvarez" y "La sangre de la uva". Tal como se expuso en el apartado anterior, la terminología vitivinícola está presente en toda la novela y por tanto se ha extraído de la totalidad de la misma, sin seleccionar partes ni capítulos concretos. No obstante, la distribución del lenguaje vitivinícola no es homogénea, lo que responde a las características de la diégesis y a la progresión de la trama argumental. Los capítulos que presentan la historia del protagonista en forma de analepsis - en concreto, el capítulo 8 y la segunda y tercera parte de la novela - se centran en la progresiva implicación de Josep en los acontecimientos políticos de la época, por lo que están prácticamente exentos de la terminología que nos ocupa. Por otro lado, a medida que el viticultor progresa en su labor y amplía su viñedo y su actividad, encontramos fragmentos más complejos y una mayor profusión de terminología, especialmente a partir del capítulo 46.

Comenzaremos presentando los términos agrupados por campos semánticos o categorías gramaticales:

\begin{tabular}{|c|c|c|}
\hline EN & DE & ES \\
\hline Vineyard & $\begin{array}{l}\text { Weinberg, Berg, } \\
\text { Weingut, Weingarten }\end{array}$ & viñedos, viña \\
\hline vines, grapevines & $\begin{array}{l}\text { Stöcke, Rebstöcke, } \\
\text { Reben, Weinblatt }\end{array}$ & vides, vid, parras \\
\hline grape, berries & Traube, Beeren & uva, grano \\
\hline Prunnings & Abschnitte & sarmientos podados \\
\hline Crop & Ernte & Cosecha \\
\hline Raisin & Rosine & Grano \\
\hline winery, bodega & $\begin{array}{l}\text { Weingut, Weinberg, } \\
\text { Winzerei }\end{array}$ & bodega, viñedo \\
\hline reds, red wines & Rote, rote Weine & vinos, vino tinto \\
\hline Vintages & Kreationen, Jahrgang & Cosechas \\
\hline plantings of vines, rows & Reihen & Hileras \\
\hline varieties of grapes & Rebensorten & variedades de uva \\
\hline Grapevine & Rebsorte & Cepa \\
\hline Tendrils & Ranken & Zarcillos \\
\hline bumps, buds & Knospen & Yemas \\
\hline shoots, cuttings & Schösslinge & Sarmientos \\
\hline
\end{tabular}


La terminología vitivinícola en The Winemaker y su traducción...

\begin{tabular}{|l|l|l|}
\hline harvest, harvesting & Ernte & $\begin{array}{l}\text { cosecha, recolección, } \\
\text { vendimia }\end{array}$ \\
\hline Roots & Wurzeln & Raíces \\
\hline clusters, bunches & Traubenbüschel, Büschel & Racimos \\
\hline pips, seeds & Kerne, Samen & Pepitas \\
\hline Stems & Stiele, Stängel & tallos, peciolos \\
\hline bulk wine & Wein & vino a granel \\
\hline reds, red wine & Rote, Rotwein & vinos, vino tinto \\
\hline must, mash & Most samt Trester, Brei & Mosto \\
\hline Yeasts & Hefen & Levaduras \\
\hline Skins & Häute & Pieles \\
\hline sampling, tasting & Verkostung & muestra, cata \\
\hline Pressing & Pressung & Prensar \\
\hline Terroir & Bodenbeschaffenheit & Terreno \\
\hline skin of wine, wineskin & Weinschlauch, Schlauch & bota de vino \\
\hline Casks & Fässer & barriles, toneles \\
\hline barrels, barrel room & Fässer & Barriles \\
\hline wine press & Weinpresse & prensa de vino \\
\hline vats, fermenting vats & Bottiche, Gärbottiche & $\begin{array}{l}\text { cubas } \\
\text { fermentación }\end{array}$ \\
\hline Plow & & Arado \\
\hline Basket & Pflug & Cesta \\
\hline Tumbrel & Korb & Carreta \\
\hline Cistern & Karre & Cisterna \\
\hline & Trog & \\
\hline
\end{tabular}

Tabla 1: partes de la vid y del viñedo

La Tabla 1 recoge los términos relacionados con la vid y la viña, siguiendo el orden de aparición en el texto y agrupando aquellos utilizados como sinónimos para facilitar la visualización. Resulta llamativo que los términos "bodega", "viña", "viñedos" y sus equivalentes se utilicen de forma indistinta en los tres idiomas. En el texto inglés encontramos el préstamo "bodega" en un total de 8 ocasiones, pero en este caso siempre referido a un establecimiento de elaboración y venta de vino.

En la versión alemana, las características propias de la composición en esta lengua propician que los términos aparezcan tanto en su forma compuesta (Weinberg, Rebstöcke, Traubenbüschel) como simplificados únicamente con el término rector (Berg, Stöcke, Büschel).

También es de señalar alguna imprecisión en la traducción de ciertos 
términos como bulk wine (traducido al alemán únicamente como Wein), reds generalizado en español como "vinos" o terroir", generalizado en español como "terreno". Por su parte, en alemán se produce una amplificación del término "mosto", añadiendo samt Trester ("junto con el orujo") para resolver la polisemia del término, que se refiere tanto al zumo de la uva como al producto del prensado que incluye residuos sólidos. En algunos casos se produce un calco, probablemente por desconocimiento de la terminología específica, como en la traducción de skins por "pieles" en lugar de "hollejos".

A continuación, se enumeran los verbos del ámbito de la vitivinicultura:

\begin{tabular}{|c|c|c|}
\hline EN & $\overline{D E}$ & ES \\
\hline Prune & Stutzen & Podar \\
\hline Harvest & Ernten & cosechar, recolectar \\
\hline clear weeds & Jäten & Desbrozar \\
\hline Weed & $\begin{array}{l}\text { die Finger in den } \\
\text { Lehm graben }\end{array}$ & $\begin{array}{lll}\text { arrancar las malas } \\
\text { hierbas }\end{array}$ \\
\hline grow (grapes) & $\begin{array}{l}\text { Anbau von Reben, } \\
\text { anbauen }\end{array}$ & cultivo de uvas, cultivar \\
\hline tend & bestellen, pflegen & cultivar, cuidar \\
\hline Plant & pflanzen, bepflanzen & Plantar \\
\hline fashion (wines) & Erzeugung von Wein & elaborar vino \\
\hline clear the land & das Land roden & despejar la tierra \\
\hline thin (vines) & Ausdünnen & Recortar \\
\hline tread, mash, stomp, plod & treten, stampfen & Pisar \\
\hline Destem & Entstielen & cortar los tallos \\
\hline Pick & Pflücken & recoger, vendimiar \\
\hline plough, plow & $\begin{array}{l}\text { die Schar führen, } \\
\text { pflügen }\end{array}$ & Arar \\
\hline top off & Nachfüllen & rellenar, filtrar \\
\hline Graft & aufpfropfen, kreuzen & Injertar \\
\hline Cull & Auslesen & Seleccionar \\
\hline Run & Bestellen & Manejar \\
\hline
\end{tabular}

Tabla 2: verbos

$8 \mathrm{Vid}$. Definición disponible en línea en: http://www.diccionariodelvino.com/index.php/terroir/ "Terroir: Término francés para describir una determinada zona geográfica que goza de unas características geológicas, climáticas, ambientales, etc. específicas que lo hacen diferente de otras zonas". 
La terminología vitivinícola en The Winemaker y su traducción...

En esta categoría gramatical se ha detectado un error de sentido en la versión alemana, en la que se ha omitido el verbo weed en el siguiente fragmento:

\begin{tabular}{|l|l|}
\hline \multicolumn{1}{|c|}{ Ejemplo 1 } \\
\hline \multicolumn{1}{|c|}{ EN } & \multicolumn{1}{|c|}{ DE } \\
\hline $\begin{array}{l}\text { Weeding on his hands and knees, } \\
\text { Josep dug his fingers into the warm } \\
\text { pebbled clay, feeling its welcome } \\
\text { grittiness under his nails. }\end{array}$ & $\begin{array}{l}\text { seine Finger in den warmen, } \\
\text { steinigen Lehm grub und seine } \\
\text { willkommene Sandigkeit unter den } \\
\text { Nägeln spürte. }\end{array}$ \\
\hline
\end{tabular}

Encontramos igualmente varias transposiciones de verbos en sintagmas nominales, tanto en alemán como en español: cortar las malas hierbas, Anbau von Reben, cultivo de wvas, Erzeugung von Wein, cortar los tallos (en lugar del término habitual "despalillar"). Del mismo modo, en lengua alemana son frecuentes las colocaciones de sustantivos con un verbo determinado: eine Ernte abwerfen (producir una cosecha), einen Jahrgang erzengen (producir una añada), Wein berstellen (elaborar vino).

Por otro lado, hallamos numerosos términos en torno a los oficios relacionados con el vino:

\begin{tabular}{|l|l|l|}
\hline \multicolumn{1}{|c|}{ EN } & \multicolumn{1}{|c|}{ DE } & \multicolumn{1}{c|}{ ES } \\
\hline Pickers & Pflücker & Recolectores \\
\hline $\begin{array}{l}\text { winemaker, wine- } \\
\text { makers, vintners, } \\
\text { makers of wine }\end{array}$ & $\begin{array}{l}\text { Winzer, Weinmacher, } \\
\text { Weinhersteller }\end{array}$ & $\begin{array}{l}\text { vinatero, hacer vino, } \\
\text { viticultores }\end{array}$ \\
\hline Peon & Arbeiter & Peón \\
\hline Cellarman & im Keller arbeiten & Bodeguero \\
\hline $\begin{array}{l}\text { grape grower, grower } \\
\text { of grapes }\end{array}$ & Winzer, Weinbauer, & $\begin{array}{l}\text { campesino, talento para } \\
\text { la uva }\end{array}$ \\
\hline $\begin{array}{l}\text { pagesos, peasant grape } \\
\text { growers }\end{array}$ & $\begin{array}{l}\text { pageses, Wein züchtende } \\
\text { Kleinbauern }\end{array}$ & $\begin{array}{l}\text { payeses, cultivadores de } \\
\text { viñas }\end{array}$ \\
\hline wine buyer & Weinaufkäufer & comprar vino \\
\hline Husbandman & Bauer & Agricultor \\
\hline
\end{tabular}

Entre ellos se encuentra el préstamo parcialmente naturalizado de 
"payeses", al cual se yuxtapone una definición en los tres idiomas. Los préstamos del catalán, español e incluso francés son frecuentes a lo largo de la novela y aparecen en el texto inglés sin cursiva y parcialmente naturalizados; no así en el alemán, donde sí identificamos la cursiva y la ortografía original:

\begin{tabular}{|l|l|l|}
\hline \multicolumn{1}{|c|}{ EN } & \multicolumn{1}{c|}{ DE } & \multicolumn{1}{c|}{ ES } \\
\hline Chateaus & Châteaux & Castillos \\
\hline grand bodegas & prächtige Höfen & grandes bodegas \\
\hline Casas & Cases & Casitas \\
\hline Padre & Pare & Padre \\
\hline $\begin{array}{l}\text { vin ordinaire, common } \\
\text { wine }\end{array}$ & $\begin{array}{l}\text { vin ordinaire, ein } \\
\text { gewöhnlicher Wein }\end{array}$ & $\begin{array}{l}\text { vin ordinaire, un vino } \\
\text { común }\end{array}$ \\
\hline Masia & Masia & Masía \\
\hline Placa & Plaça & Plaza \\
\hline Cru & Cru & Cru \\
\hline Garnacha & Garnatxa & Garnacha \\
\hline Cariñena & Carinyena & Cariñena \\
\hline Ull de Llebre & Ull de Llebre-Trauben & Tempranillo \\
\hline
\end{tabular}

Tabla 4: préstamos

La tabla 4 muestra el diverso tratamiento que se da a los préstamos en los tres textos. La versión alemana es la más exhaustiva y consistente en el uso de la cursiva en los préstamos, además de incluir un glosario al final de la novela con todos los préstamos o culturemas y sus definiciones, como se puede ver en los siguientes ejemplos:

Cru (franz.): Gewächs. In Frankreich eine Prädikatsbezeichnung für höher klassifizierte Regionen und deren Weine.

Garnatxa: katalanischer Begriff für die rote spanische Traube Garnacha, die im deutschen Sprachraum Grenache genannt wird. Kommt ursprünglich aus Aragonien, wird heute im gesamten westlichen Mittelmeerraum angebaut. In Spanien vor allem im Anbaugebiet Priorat.

Phylloxera: Die Reblaus (Viteus vitifoliae) ist eine Pflanzenlaus aus der Familie der Zwergläuse (Phylloxeridae), die an den Wurzeln des Rebstocks saugt, wodurch die Rebe nachhaltig geschädigt wird. 
La terminología vitivinícola en The Winemaker y su traducción...

Cabe señalar que el texto alemán emplea la minúscula en los préstamos en cursiva, mientras en otros prescinde de la cursiva y mayusculiza la inicial de los sustantivos, siguiendo la convención habitual de la lengua alemana: Garnatxa, Pare, Governador. Estos últimos corresponden a los nombres propios como variedades de uva, cargos o instituciones.

Asimismo, es de destacar la abundancia de adjetivos y valoraciones sobre las propiedades y la calidad del vino y la vid:

\begin{tabular}{|l|l|l|}
\hline \multicolumn{1}{|c|}{ EN } & \multicolumn{1}{c|}{ DE } & \multicolumn{1}{c|}{ ES } \\
\hline Juicy & Saftig & Jugoso \\
\hline economical vines & karge Stöcke & reducidas vides \\
\hline decent vin ordinaire & anständigen vin ordinaire & un vin ordinaire decente \\
\hline honest wine & ehrlicher Wein & vinos honestos \\
\hline rather poor wine & ziemlich minderwertig & vino tirando a malo \\
\hline thin, sour, harsh & $\begin{array}{l}\text { dünn und sauer und } \\
\text { beißend }\end{array}$ & $\begin{array}{l}\text { aguado, amargo y } \\
\text { áspero }\end{array}$ \\
\hline $\begin{array}{l}\text { had long since lost its } \\
\text { edge }\end{array}$ & $\begin{array}{l}\text { obwohl er sein Aroma } \\
\text { schon längst verloren } \\
\text { hatte }\end{array}$ & $\begin{array}{l}\text { ya hacía tiempo que } \\
\text { estaba picado }\end{array}$ \\
\hline poor row & $\begin{array}{l}\text { entlang einer } \\
\text { armseligen Reihe }\end{array}$ & hilera mediocre \\
\hline musky, sweet scent & $\begin{array}{l}\text { den schweren, süßen } \\
\text { Geruch }\end{array}$ & $\begin{array}{l}\text { aroma almizclado y } \\
\text { dulce }\end{array}$ \\
\hline $\begin{array}{l}\text { rich and } \\
\text { fruity, yet dry enough }\end{array}$ & $\begin{array}{l}\text { reich und fruchtig und } \\
\text { doch trocken genug }\end{array}$ & $\begin{array}{l}\text { intenso y afrutado, y al } \\
\text { mismo tiempo bastante } \\
\text { seco }\end{array}$ \\
\hline
\end{tabular}

Tabla 5: adjetivos y valoraciones sobre el vino y la vid, sus cualidades o calidad

Llamaremos la atención únicamente sobre la traducción "vinos honestos", por considerar que se trata de un calco del texto inglés y que la honestidad es una cualidad difícilmente atribuible al vino. Propondríamos en su lugar la traducción propia "vinos sin pretensiones".

Por último, a partir del capítulo 46 encontramos una abundancia de fragmentos con descripciones más complejas sobre la elaboración del vino o la cata:

\begin{tabular}{|c|c|c|}
\hline \multicolumn{3}{|c|}{ Ejemplo 2} \\
\hline EN & DE & ES \\
\hline Everything in the must & Alles im Most, das & El contenido del mosto \\
\hline
\end{tabular}




\begin{tabular}{|l|l|l|}
\hline that wasn't juice-the & nicht Saft war -die & que no era puro zumo \\
skins, the seeds, and the & Häute, die Samen, die & -pieles, semillas y \\
stems-were repeatedly & Stängelreste-, trieb & tallos- flotaba en la \\
buoyed up to the & immer wieder an die & superficie del líquido y \\
surface of the liquid to & Oberflächer der & formaba una capa que \\
become a cap, and the & Flüssigkeit und bildete & enseguida se secaba. \\
cap soon dried. Every & dort eine Kappe, die & Cada pocas horas, \\
few hours Josep & schnell austrocknete. & Josep vaciaba algo de \\
drained off liquid from & Alle Paar Stunden ließ & líquido de la parte \\
the bottom of the tank & Josep Flüssigkeit von & inferior de la cuba, se \\
and climbed a ladder so & unten aus dem Bottich & subía a una escalerilla \\
he could pour the juice & ab, um den Saft über & para poder derramarlo \\
over the floating solids, & die oben schwebenden & por encima de los \\
and now and again he & Feststoffe zu gießen, residuos sólidos que \\
would use a rake to & und hin und wieder & flotaban y, de vez en \\
push the cap down, & stieß er die Kappe auch & cuando, usaba un \\
mixing it with the body & mit einem Rechen nach & rastrillo para empujar la \\
of the liquid. & unten, um sie aufs & capa hacia el fondo y \\
Neue mit der & mezclarla con el \\
Flüssigkeit zu mischen. & líquido.
\end{tabular}

El ejemplo 2 presenta una parte del proceso de la elaboración del vino, el remontado (extracción del mosto por la parte inferior del depósito para reintroducirlo por arriba) o bazuqueo (romper el sombrero de forma manual con la ayuda de una vara o bazuqueador para que se mezcle con el mosto) ${ }^{9}$. Observamos algunas imprecisiones terminológicas, como la ya comentada "pieles" (en lugar de "hollejos") o la traducción de cap por "capa" en lugar de "sombrero".

En el siguiente ejemplo, se describe la plaga de filoxera que asoló los viñedos franceses y españoles en el s. XIX:

\begin{tabular}{|l|l|l|l|}
\hline \multicolumn{4}{|c|}{ Ejemplo 3 } \\
\hline \multicolumn{1}{|c|}{ EN } & \multicolumn{3}{|c|}{ DE } \\
\hline $\begin{array}{l}\text { The plague came to } \\
\text { Europe in grapevines }\end{array}$ & $\begin{array}{l}\text { Die Seuche kam mit } \\
\text { Stöcken aus Amerika }\end{array}$ & $\begin{array}{l}\text { La plaga llegó a } \\
\text { Europa en parras }\end{array}$ \\
\hline
\end{tabular}

${ }^{9}$ Vid. explicación de este proceso disponible en línea en: https://vivancoculturadevino.es/blog/2016/06/14/proceso-de-elaboracion-del-vino-tinto/ 
La terminología vitivinícola en The Winemaker y su traducción...

\begin{tabular}{|c|c|c|}
\hline $\begin{array}{l}\text { imported from } \\
\text { America, but there is an } \\
\text { American grapevine } \\
\text { whose roots the aphids } \\
\text { don't eat. Perhaps the } \\
\text { roots contain an } \\
\text { element poisonous to } \\
\text { the aphids, or maybe } \\
\text { they simply taste very } \\
\text { bad. When cuttings of } \\
\text { our doomed vines are } \\
\text { grafted onto these } \\
\text { American roots, the } \\
\text { aphids don't bother } \\
\text { them. }\end{array}$ & $\begin{array}{l}\text { nach Europa, aber es gibt } \\
\text { eine amerikanische } \\
\text { Rebsorte, deren Wurzeln } \\
\text { diese Laus nicht frisst. } \\
\text { Vielleicht enthalten die } \\
\text { Wurzeln etwas, das für } \\
\text { die Läuse giftig ist, oder } \\
\text { vielleicht schmecken sie } \\
\text { einfach nur sehr schlecht. } \\
\text { Wenn Schösslinge } \\
\text { unserer zum Untergang } \\
\text { verurteilten Reben auf } \\
\text { diese amerikanischen } \\
\text { Wurzeln aufgepfropft } \\
\text { werden, können die } \\
\text { Läuse ihnen nichts tun. }\end{array}$ & 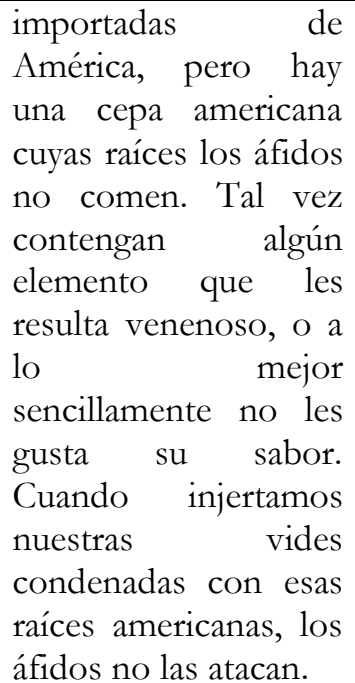 \\
\hline
\end{tabular}

En el ejemplo 3, la traducción de la terminología es precisa a excepción de la omisión de cuttings ("sarmientos") en la versión española.

\section{Conclusiones}

En los tres textos que nos ocupan se ha podido observar una profusión de terminología vitivinícola, así como información específica sobre el cultivo de la vid, la elaboración del vino y otros procesos relacionados. La traducción de dicha terminología es, por lo general, adecuada en los idiomas analizados, con la salvedad de algunos calcos o elisiones. La precisión en el uso de algunos términos, su número (singular o plural) y su ortografía, sin embargo, presenta variaciones en los tres idiomas, sin que se vea alterado el sentido del texto ni el efecto estético que este produce. En concreto, la abundancia de sinónimos en el lenguaje especializado es una constante en los tres idiomas. Esto nos lleva a concluir que existe una mayor flexibilidad en el uso de la terminología especializada en el texto literario que en el texto especializado, si bien es preferible que exista la mayor homogeneidad posible en lo tocante al uso de la cursiva o a la naturalización de los préstamos. En este sentido, el texto alemán es el más cuidado tanto en su ortografía como en el tratamiento de los préstamos, y el glosario añadido al final del libro contribuye a la experiencia estética e informativa del lector.

El lenguaje especializado que hemos analizado tiene la función de contextualizar la obra, caracterizar a los personajes y a sus actividades y 
MARÍA Pilar CASTiLlo Bernal

sumergir al lector en la trama y en la evolución del protagonista. Por otro lado, resulta enormemente informativo en lo tocante a las circunstancias geográficas e históricas descritas, como son la época de mediados del s. XIX en los viñedos del Penedés, las relaciones comerciales con Francia o las plagas que asolaron la región. Todo ello sirve asimismo el propósito de promocionar el producto vinícola y su zona de origen, tal como declara el mismo autor en los agradecimientos de las tres versiones analizadas. Por otro lado, no es posible confirmar o descartar que la versión en lengua inglesa sea la original, aunque los paratextos encontrados así parecen indicarlo.

Por todo lo anterior, consideramos que The Winemaker presenta un interesante caso de confluencia del lenguaje especializado y el literario, y que como tal bien puede servir de introducción al estudio de ambos. Con nuestro trabajo esperamos haber contribuido a la divulgación y mejor comprensión de esta novela en el ámbito hispanohablante.

\section{Referencias bibliográficas}

Balbuena TorezAnO, M. Carmen; Álvarez Jurado, Manuela (eds.), Lenguajes especializados y traducción: Vitivinicultura. Córdoba: EDF, 2015.

CAstillo Bernal, María Pilar, "Las paremias en torno al campo semántico del vino en alemán y su traducción al español”. En: Estudios Franco-Alemanes, 4, 2012, 57-71.

CORBACHO SÁNCHEZ, Alfonso, "Apuntes sobre fraseología en torno al vino como componente base en las lenguas alemana y española: un estudio lingüístico contrastivo". En: Babel afilial: Aspectos de filología inglesa y alemana, 13, 2004, 111-128.

DICCIONARIO DEL VINO. Disponible en línea en: http://www.diccionariodelvino.com/ [Fecha de consulta: 9 de agosto 2017].

ESCOBAR MORENO, Julia, “"Experiencias reales». Identificación y resolución de problemas documentales en la traducción de textos narrativos". En: Gonzalo García, Consuelo/ García Yebra, Valentín (eds.): Manual de documentación para la traducción literaria. Madrid: Arco Libros, 2005, 285-295.

FORGAS I BERDET, Esther, Los ciclos del pan y el vino en las paremias hispanas. Madrid: Ministerio de Agricultura, Pesca y Alimentación, Centro de Publicaciones, 1996.

GORDON, Noah, El último judío. Traducción de María Antonia Menini. Barcelona: Roca Editorial de Libros, S.L., 2011.

Gordon, Noah, La bodega. Traducción de Enrique de Hériz. Barcelona: Roca Editorial de Libros, S.L., 2007.

Gordon, Noah, Der Katalane. Traducción de Klaus Berr. Múnich: Wilhelm 
La terminología vitivinícola en The Winemaker y su traducción...

Heyne Verlag, 2008.

GORDON, Noah, The Winemaker. Barcelona: Barcelona Digital Editions, S.L, 2012.

GORDON, Noah, página web oficial. Disponible en línea en:

http://www.noahgordonbooks.com/index.html [Fecha de consulta: 9 de agosto 2017]

IbÁÑEZ RodríGueZ, Miguel et al. (eds.), El lenguaje de la vid y el vino y su traducción. Valladolid: Universidad de Valladolid, Secretariado de Publicaciones e Intercambio Editorial, 2006.

IBÁÑEZ RODRÍGUEZ, Miguel et al. (eds.), Vino, lengua y traducción. Valladolid: Universidad de Valladolid, Secretariado de Publicaciones e Intercambio Editorial, 2010.

MARTíneZ Fraile, Cristina, "Los atributos subjetivos asociados al vino presentes en las expresiones fijas alemanas y las españolas. Una aproximación intercultural”. En: Balbuena Torezano, M. Carmen y Álvarez Jurado, Manuela (eds.), Lenguajes especializados y traducción: Vitivinicultura. Córdoba: EDF, 2015, 133-144.

Muro MuniLla, Miguel Ángel, El cáliz de letras: historia del vino en la literatura. Briones (La Rioja): Fundación Dinastía Vivanco, 2006.

NORD, Christiane, "Donde no hay vino no hay amor: el vino en la literatura". En: Ibáñez Rodríguez, Miguel et al. (eds.), Vino, lengua y traducción. Valladolid: Universidad de Valladolid, Secretariado de Publicaciones e Intercambio Editorial, 2010, 193-203.

Roca Editorial De Libros, S.L., "El último judío". Disponible en línea en: http://www.rocalibros.com/rocaeditorial/catalogo/Noah+Gordon/El+ultimo+judio [Fecha de consulta: 9 de agosto 2017]

SANDRINI, Peter, Terminologiearbeit im Recht. Deskriptiver begriffsorientierter Ansatz vom Standpunkt des Übersetzers. Viena: International Network for Terminology, 1996.

SANTANA LÓPEZ, Belén, FORTEA, Carlos, "El hombre de las mil y una caras: el traductor literario como gestor experto de fuentes documentales especializadas". En: Santana López, Belén / Travieso Rodríguez, Críspulo (eds.): Puntos de encuentro: los primeros 20 años de la Facultad de Traducción y Documentación de la Universidad de Salamanca. Salamanca: Ediciones Universidad de Salamanca, 2013, 135-150.

SOLANA, Maite, "Las fuentes documentales en la práctica de la traducción literaria". En: Gonzalo García, Consuelo/ García Yebra, Valentín (eds.): Manual de documentación para la traducción literaria. Madrid: Arco, 2005, 247-266. 
María Pilar Castillo Bernal

VIVANCO, “¿Cómo es el proceso de elaboración del vino tinto?” Disponible en línea en: https://vivancoculturadevino.es/blog/2016/06/14/proceso-deelaboracion-del-vino-tinto/ [Fecha de consulta: 9 de agosto 2017]

ZARANDONA Fernández, Juan $\mathrm{M}$, “Novelas populares norteamericanas ambientadas en viñedos y bodegas y algunas reflexiones de traducción". En: Ibáñez Rodríguez, Miguel et al. (eds.), Vino, lengua y traducción. Valladolid: Universidad de Valladolid, Secretariado de Publicaciones e Intercambio Editorial, 2010, 205-225. 